[13] H. Lebesgue, Sur la représentation trigonométrique approchée des fonotions satisfaisant $a$ une condition de Lipschitz, Bull. Soc. Math. France 38 (1910), 184-210.

[14] G. G. Lorentz, Inequalities and the saturation of Bernstein polynomials, in: On Approximation Theory (Proc. Conf. Oberwolfach 1963, Eds. P. I. Butzer, J. Korevaar), ISNM 5, Birkhäuser, Basel 1964, 200-207.

[15] W. Orlicz, Über Folgen linearer Operationen, die von einem Parameter abhängen, Studia Matl. 5 (1934), 160-170.

[16] K. I. Oskolkov, An estimate of the rate of approximation of a continuous function and its conjugate by Fourier sums on a set of lotal measure (Russian), Iav. Acad. Nauk SSSR Ser. Mat. 38 (1974), 1393-1407 [Math. USSR-Izv. 8 (1974), 13721386].

[17] - Lebesgue's inequality in a uniform metrio and on a set of full measure (Russian), Mat. Zametki 18 (1975), 515-526 [Math. Notes 18 (1975), 895-902].

[18] A. F. Timan, Theory of Approximation of Functions, Pergamon Pross, Now York 1963.

[19] P. O. H. Vértesi, On the almost everywhere divergence of Lagrange inlerpolation (Complex and trigonometric cases), Acta Math. Acad. Sci. Hungar. (to appear).

[20] K. Zeller, FK-Räume in der Funktionentheorie I, Math. Z. 58 (1953), 288-305.

\section{On the extension of \\ continuous linear maps in function spaces and the splitness of Dolbeaut complexes of \\ holomorphic Banach bundles}

by

NGUYEN VAN KHUE (Warszawa)

Abstract. The paper investigates the extension of continuous linear maps with values in the spaces of sections of coherent analytic sheaves over analytic spaces. It is shown that the space $H^{0}(X, \mathscr{P})$, where $\mathscr{S}$ is a coherent analytic sheaf over a paracompact an alytic space $X$ has the extension property with respect to the class of

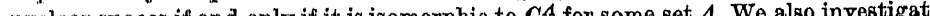
the existence of continuous linear projections of the space $O_{\xi}^{\circ}(R(X))$ onto $C_{\xi}(X)$, where $R(X)$ is the regular part of $X$ and $\xi$ is a holomorphic Banach bundle over $X$. The splitness of Dolbeauti complexes of holomorphic Banach bundles over complex manifolds is considered. We prove that on complex manifolds which are increasing unions of open Stein sots these complexes split only at positive dimensions.

Introduction. In the present paper we consider extensions of continuous linear maps with values in some function spaces of complex analysis and the splitness of Dolbeaut complexes of holomorphic Banach bundles over complex manifolds. These problems have been investigated by soveral authors ([6], [8]). The paper contains three sections.

In $\S 1$ we prove that the space $H^{0}(X, \mathscr{S})$ has the extension property with respect to the class of $s$-nuclear spaces if and only if it is isomorphic to $C^{\wedge}$ tor some set $A$.

Section $\$ 2$ is devoted to the study of the existence of continuous linear projections of $O_{\xi}^{\infty}(R(X))$ onto $\mathcal{O}_{\xi}(X)$. It is shown that when $X$ is Stoin such a projoction exists if and only if $X$ is discrete.

In $\$ 3$ wo investigato the splitness of Dolbeaut complexes of holomorphic Banach bundles over complex manifolds. We prove that on complex manifolds which are increasing unions of open Stein sets these complexes split only at positive dimensions. Let us note that the splitness of Dolbeaut complexes of holomorphic vector bundles over Stein manifolds has been established by Palamodor ([8]). 
Acknowledgment. I wish to express my gratitude to Professor W. $1 / \mathrm{se-}$ lazko for his guidance and constant encouragment during the preparation of this paper.

\$ 1. The extension of continuous linear maps with values in $H^{0}(X, \mathscr{P})$. Let $E$ be a locally convex space. By $\mathscr{U}(E)$ we denote the set of all balinced convex neighbourhoods of zero in $E$. For each $U \in \mathscr{U}(E)$ let $\varrho_{U}$ denote the Minkowski functional on $E$ of $U$ and let $E(U)$ be the completion of $E / \varrho_{U}=E / \varrho_{U}^{-1}(0)$ equipped with the norm $\varrho_{U}$. The canonical map from $D$ into $E(U)$ is denoted by $\pi(U)$ and from $D(V)$, where $V \in \mathscr{U}(\mathbb{V}), V \subset U$, into $E(U)$ by $\omega(V, \dot{U})$. Finally by $E^{\prime}$ we denote the strongly dual space of $D$.

The space $E$ is called $s$-nuclear $([9])$ if for each $U \in \mathscr{U}(D)$ thoro oxistis a $V \in \mathscr{U}(E)$ such that $V \subset U$ and the map $\omega(V, U)$ is $s$-nuelear, i.e. $\omega(V, U)$ can be represented in the form

$$
\omega(V, U) u=\sum_{j=1}^{\infty} \lambda_{j} u_{j}^{\prime}(u) v_{j},
$$

where

$$
\begin{aligned}
& \lambda_{1} \geqslant \lambda_{2} \geqslant \ldots>0, \\
& \sum_{j=1}^{\infty} \lambda_{j}^{p}<\infty \quad \text { for } \quad p>0, \\
& u_{j}^{\prime} \in E(V)^{\prime}, \quad v_{j} \in E(U), \quad \sup \left\{\left\|u_{j}^{\prime}\right\|,\left\|v_{j}\right\|\right\}<\infty .
\end{aligned}
$$

Let $X$ be a paracompact analytic space and lot $\mathscr{S}$ be a coherent analytic sheaf over $X$. It is known ([4]) that $H^{0}(W, \mathscr{S})$ has a unique locally convex space structure, where $W$ is an open set in $X$, such that the restiction maps are continuous. In this section we prove the following

THeOREM 1.1. Let $X$ be a paracompact analytic space and let $\mathscr{P}$ be a coherent analytic sheaf over $X$. Then the following conditions are equivalent:

(i) $H^{0}(X, \mathscr{S}) \cong \boldsymbol{C}^{\Lambda}$ for some set $\Lambda$.

(ii) Every continuous linear map from every subspace of every locally convex space into $H^{0}(X, \mathscr{S})$ can be extended to a continuous linear map.

(iii) The statement (ii) holds for all subspaces of all s-nuolear spaces.

Proof. (i) $\Rightarrow$ (ii) follows from the Hahn-Banach thoorom.

(ii) $\Rightarrow$ (iii) is trivial.

(iii) $\Rightarrow$ (ii) is an immediate consequence of Propositions 1.2 and 1.3.

Proposirion 1.2 ([6]). Let $X$ be a paracompact analytic space and let $\mathscr{S}$ be a coherent analytic sheaf over $X$. Then $H^{0}(X, \mathscr{S})$ is s-nuclear.

Propositron 1.3. Let $E$ be an s-nuclear subspace of a locally conveco space F. Then there exist an s-nuclear space $\tilde{B}$ and continuous linear maps $\tilde{i}$ : $\pi \rightarrow \tilde{B}$ and $h: T \rightarrow \tilde{E}$ such that

$$
h i=\tilde{i} \text {. and } \tau_{i} i s \text { an embedding. }
$$

I. Iro $i$ donotos the canonical embedding of $E$ into $T$.

Wo nood the following

Liwm 1.4 ([6]). Every s-nucilear map from a subspace of a Banach space into a Banach space can be extended to an s-nuclear map.

Lismma 1.5 ([6]). If or every s-nuctear map $T$ from a Barbach space $A$ into a Banach space $B$ there exist s-nuclear maps $P: A \rightarrow O$ and $Q: O \rightarrow B$ such that $T=Q P$.

Lamma 1.6 ([6]). Tet $\alpha: A \rightarrow B$ and $\beta: B \rightarrow O$ be continuous linear maps between Banach spaces such that ia is s-nuclear for some embedding $i$ and $\beta$ is quasimnolear. Then $\beta \alpha$ is s-nuclear.

Proof of Proposition 1.3. Let $U, V \in \mathscr{U}(\mathbb{E})$. We write $U<V$ if $U \supset V: \tilde{V} \cap D$, whero $\tilde{V} \in \mathcal{U}(F)$ and $\omega(V, U)$ is $s$-nuclear. Let $U, V \in \mathcal{U}(E)$ and $U<V$. By temmas 1.4. and 1.5 there exist sequences $\left\{P_{j}(V, U)\right\}$ and $\left\{Q_{j}(V, U)\right\}$ of $s$-muclear maps such that

$$
\begin{aligned}
& \tilde{\omega}(V, U)=Q_{1}(V, U) P_{1}(V, U), \\
& P_{j}(V, U)=\dot{Q}_{j+1}(V, U) P_{j+1}(V, U),
\end{aligned}
$$$$
\ldots \ldots \ldots \ldots
$$

whore $\tilde{\omega}(V, U): I^{N}(\tilde{V}) \rightarrow E(U)$ is an s-nuclear extension of $\omega(\nabla, U)$. Fix $\tilde{W} \in \mathscr{C}\left(F^{\prime}\right)$. Lut $\mathscr{F}(\tilde{W})$ denote the set of ull finite sequences

$$
a=\left\{\left(\tilde{V}_{1}, \tilde{U}_{1}\right), \ldots,\left(\tilde{V}_{n}, \tilde{U}_{n}\right) ; k_{1}, \ldots, k_{n}\right\},
$$

whoro

$$
\begin{array}{r}
\tilde{V}_{j}, \tilde{U}_{j} \in \mathscr{U}(F), \quad \tilde{W} \subset \tilde{V}_{j} \subset \tilde{U}_{j}, \quad U_{j}=\tilde{U}_{j} \cap E<\nabla_{j}=\tilde{V}_{j} \cap E \\
\text { and } \quad \eta_{i j} \in\{1,2, \ldots\}, j=1, \ldots, n .
\end{array}
$$

For $a_{j} \ldots\left\{\left(\tilde{V}_{1}^{j}, \tilde{U}_{1}^{j}\right), \ldots,\left(\tilde{V}_{n_{j}}^{j}, \tilde{U}_{n_{j}}^{j}\right), k_{1}^{j}, \ldots, 7_{n_{j}}^{j}\right\} \in \mathscr{F}(\tilde{W}), j=1,2$ we write $a_{1}<a_{2}$ iff

$$
\left\{\left(V_{1}^{1}, U_{1}^{1}\right), \ldots,\left(V_{u_{1}}^{1}, \tilde{U}_{n_{1}}^{1}\right)\right\} \subset\left\{\left(\tilde{V}_{1}^{2}, U_{1}^{2}\right), \ldots,\left(\tilde{V}_{n_{2}}^{2}, \tilde{U}_{n_{2}}^{2}\right)\right\} .
$$

For exch $a \in \mathscr{W}(\tilde{W})$ by $\tilde{E}(\tilde{W}, a)$ wo denoto the space $F(\tilde{W}) / \varrho_{a}$ equipped with tho norm

$$
\varrho_{a}(u)=\sum_{j=1}^{n}\left\|P_{k_{j}}\left(V_{j}, U_{j}\right) \omega\left(\tilde{W}, \tilde{V}_{j}\right) u\right\| .
$$

Obviously, if $a_{1}<a_{2}$, then the indentity map id: $F(\tilde{W}) \rightarrow F(\tilde{W})$ induces a continuous linear map $\tilde{\omega}\left(\tilde{W}, a_{2}, a_{1}\right): \tilde{W}\left(\tilde{W} ; a_{2}\right) \rightarrow \tilde{W}\left(\tilde{W} ; a_{1}\right)$ and 
$\tilde{\omega}\left(\tilde{W}, a_{3}, a_{1}\right)=\tilde{\omega}\left(\tilde{W}, a_{3}, a_{2}\right) \tilde{\omega}\left(\tilde{W}, a_{2}, a_{1}\right)$ for $a_{3}>a_{2}$. Thus we can put

$$
\tilde{E}(\tilde{W})=\lim _{\leftarrow}\{\tilde{E}(\tilde{W}, a), \tilde{\omega}(\tilde{W}, a, b)\} .
$$

From the construction of $\tilde{\boldsymbol{E}}(\tilde{W})$ it follows that if $\tilde{W}_{1}, \tilde{W}_{2} \in \mathscr{U}(\boldsymbol{F}), \tilde{W}_{2} \subset \tilde{W}$ then the map $\omega\left(\tilde{W}_{2}, \tilde{W}_{1}\right)$ induces a continuous linear map $\tilde{\omega}\left(\tilde{W}_{2}, \tilde{W}_{1}\right)$ : $\tilde{E}\left(\tilde{W}_{2}\right) \rightarrow \tilde{E}\left(\tilde{W}_{1}\right)$ such that

$$
\Pi\left(\tilde{W}_{1}\right) \omega\left(\tilde{W}_{2}, \tilde{W}_{1}\right)=\tilde{\omega}\left(\tilde{W}_{2}, \tilde{W}_{1}\right) h\left(\tilde{W}_{2}\right)
$$

where $h\left(\tilde{W}_{j}\right): I\left(\tilde{W}_{j}\right) \rightarrow \tilde{E}\left(\tilde{W}_{j}\right)$ denotes the canonical nap. Thus putting

$$
\tilde{\boldsymbol{E}}=\lim _{\leftarrow}\left\{\tilde{\boldsymbol{E}}(\tilde{\boldsymbol{W}}), \tilde{\omega}\left(\tilde{\boldsymbol{W}}, \tilde{W}^{\prime}\right)\right\}, \quad h=\lim _{\leftarrow} h(\tilde{W}) \text { and } \tilde{\imath}=h i,
$$

we get a locally convex space $\tilde{E}$ and continuous linoar maps $\tilde{\imath}: U \rightarrow \tilde{H}$ and $h: F \rightarrow \tilde{E}$ such that $h_{\iota}=\tilde{i}$. Honce to finish the proof it remains to ostablish the following:

(a) $i$ is an embedding.

(b) $\tilde{\boldsymbol{E}}$ is s-nuclear.

Proof of (a). Let $\tilde{\imath}\left(u_{\alpha}\right) \rightarrow 0$ and let $U, V \in \mathscr{U}(E)$ be such that $U<V$. Applying (1.3) to $a=(V, U, 1)$, we havo

$$
\begin{aligned}
\lim \varrho_{U}\left(u_{\alpha}\right) & =\lim \left\|\tilde{\omega}(V, U) \pi(\tilde{V}) u_{a}\right\| \\
& =\lim \left\|Q_{1}(V, U) P_{1}(V, U) \pi(\tilde{V}) u_{a}\right\|=0 .
\end{aligned}
$$

Hence $\mathscr{U}_{\alpha} \rightarrow 0$ and therefore $i$ is an cmbedding.

Pro of of (b). It suffices to show that $\tilde{E}(\tilde{W})$ is $s$-nucloar for $\tilde{W} \in \mathscr{U}\left(I^{\prime}\right)$.

Let $\tilde{W} \in \mathscr{U}\left(F^{\prime}\right)$ and let $a_{1}, a_{2} \in \mathscr{F}(\tilde{W}), a_{1}<a_{2}$. Consider the mays

$$
\begin{aligned}
& \theta_{1}: \tilde{E}\left(\tilde{W}, a_{1}\right) \rightarrow \operatorname{ImP}_{k_{1}^{1}}\left(V_{1}^{1}, U_{1}^{1}\right) \oplus \ldots \oplus \operatorname{ImP}_{k_{n_{1}}^{1}}\left(V_{n_{1}}^{1}, U_{n_{1}}^{1}\right), \\
& \theta_{2}: \tilde{E}\left(\tilde{W}, a_{2}\right) \rightarrow \operatorname{ImP}_{k_{1}^{1}}\left(V_{1}^{1}, U_{1}^{1}\right) \oplus \ldots \oplus \operatorname{ImP}_{k_{n_{1}^{1}}^{1}}\left(V_{n_{1}}^{1}, U_{n_{1}}^{1}\right)
\end{aligned}
$$

given by the formulas

$$
\theta_{1}(\bar{u})=\left(P_{k_{1}^{1}}\left(V_{1}^{1}, U_{1}^{1}\right) \omega\left(\tilde{W}, \tilde{V}_{1}^{1}\right) u, \ldots, P_{k_{n_{1}}^{1}}\left(V_{n_{1}}^{1}, U_{n_{1}}^{1}\right) \omega\left(\tilde{W}, \tilde{V}_{n_{1}}^{1}\right) u\right)
$$

where $u \in F(W), \bar{u}=u$ mod $\varrho_{a_{1}^{-1}}(0)$,

$$
\begin{gathered}
\theta_{2}(\bar{u})=\left(Q_{k_{1}^{1}+1}\left(V_{1}^{1}, U_{1}^{1}\right) P_{k_{1}^{1}+1}^{\prime}\left(V_{1}^{1}, U_{1}^{1}\right) \omega\left(\tilde{W}, \tilde{V}_{1}^{1}\right) u_{1}, \ldots\right. \\
\left.\ldots, Q_{k_{n_{1}}^{1}+1}\left(V_{n_{1}}^{1}, U_{n_{1}}^{1}\right) I_{k_{n_{1}}^{1}+1_{1}}^{1}\left(V_{n_{1}}^{1}, U_{n_{1}}^{1}\right) \omega\left(\tilde{W}, \tilde{V}_{n_{1}}^{1}\right) u_{1}\right),
\end{gathered}
$$

where $u \in F(W), \bar{u}=u \bmod \varrho_{a_{2}^{-1}}(0)$.

By (1.2) we have $\theta_{2}=\theta_{1} \tilde{\omega}\left(\tilde{W}, a_{2}, a_{1}\right)$ and by (1.3) $\theta_{1}$ is an embedding. Since $Q_{j}(V, U)$ is $s$-nuclear for $U<V$ and for $j \geqslant 1$, it follows that $\theta_{2}$ is
8 -nuclear. Tonce, by Temma 1.6, $\tilde{\omega}\left(\tilde{W}, a_{3}, a_{1}\right)$ is 8 -nuclear for $a_{2}>a_{2}$ This completes the proof of (b).

\section{(ii) $\Rightarrow$ (i) results from the following}

IFMma 1.7. Let $Q$ be a Montel space and let $\theta$ denote the canonical embedding of $Q$ into $J=I I\{Q(U): U \in \mathscr{U}(Q)\}$. Assume that there exists a continuous linear projection $T$ of $J$ onto $\operatorname{Im} \theta$. Then $Q \cong C^{\Lambda}$ for some set $A$.

Proof. Since $J=\operatorname{Im} T \oplus \operatorname{Im}(\mathrm{id}-T)$, it follows that $X$ is open. For each finite sot $a \subset q(Q)$ put

$$
G(a)=\prod_{U \in a} S(U) \times \prod_{U i a} Q(U)
$$

where $S(U)$ denotes the unit open ball in $Q(U)$. Since $T$ is open, $T$ induces a continuous linear opon map

$$
T(a): J / \varrho_{Q(a)}=\prod_{U \in a} Q(U) \rightarrow \operatorname{Im} \theta / \varrho_{T G(a)}
$$

Since $Q$ is Montel, wo infor that $T(a)$ is compact. Hence $\operatorname{dim} \operatorname{Im} \theta / \varrho_{Z G(a)}<\infty$. On the other hand, since $\{T G(a) / m\}$ forms a basis of neighbourhoods of zoro in $\operatorname{Tm} \theta$ and $\operatorname{In} \theta \simeq Q$, we have $\operatorname{dim} Q(U)<\infty$ for $U \in \mathscr{U}(Q)$.

Let $\left\{u_{a}^{\prime}: \alpha \in \Lambda\right\}$ bo a vector basis of $Q^{\prime}$. Define a continuous linear map. $\gamma: Q \rightarrow C^{\Lambda}$ by $\gamma u=\left(u_{a}^{\prime}(u)\right)$. Sinco $\varrho_{U}(u)=\sup \left\{\left|u^{\prime}(u)\right|: u^{\prime} \in U^{\circ}\right\}$, where $U^{\circ}$ is tho polur of $U$ and since $U^{\circ}$ is contained in a finite dimensional subspace of $Q^{\prime}$, it follows that $\gamma$ is an embedding. Combining this with the relation $\overline{\operatorname{Im} \gamma}=C^{4}$, wo infer that $\gamma$ is an isomorphism.

Tho lomma is proved.

$\S 2$. The existence of continuous linear projections of $C_{\xi}^{\infty}(R(X))$ onto $O_{\xi}(X)$. Lot $X$ bo a paracompact analytic space and let $\xi$ be a holomorphic Banuch bundle over $X$. By $O_{\xi}(X)$ we denote the space of holomorphic sections of $\xi$ on $X$ equipped with the compact-open topology and by $C_{\xi}^{\infty}(R(X))$ the space of $O^{\infty}$-sections of $\xi$ on $R(X)$ equipped with the topology of uniform convergence of all derivatives on compact sets in $X$. Since the restriction map $O(X) \rightarrow \mathcal{O}(R(X))$ is an embedding ([5]), it follows that $O_{\xi}(X)$ is contrined in $\partial_{\xi}^{\infty}(R(X))$ as a subspaco.

Wo prove tho following

Tumorwar 2.1. Let $X$ be a locally irreducible stein space and let $\xi$ be a holomorphio veotor bundle over $X$. Let $\mathscr{S}$ be a coherent analytic subsheaf of the sheaf $\mathcal{O}_{\xi}$ of germs of holomorphic sections of $\xi$ on $X$. Then $H^{0}(X, \mathscr{S})$ is. complemented in $O_{\xi}^{\infty}(R(X))$ if and only if $X$ is discrete.

Sinco $X$ is stoin and $\mathscr{S}$ is coherent by ([1], Lemma 3.4, p. 38), it. sufficos to prove tho following 
LEMMA 2.2. Let $X$ be a locally irreducible analytio space and let $\mathscr{S}$ and $\xi$ be as in Theorem 2.1. Then $H^{0}(X, \mathscr{S})$ is complemented in $O_{\xi}^{\infty}(R(X))$ if and only if $H^{0}(X, \mathscr{S}) \cong C^{\Lambda}$ for some set $\Lambda$.

Proof: Let $P: C_{\xi}^{\infty}(R(X)) \rightarrow H^{0}(X, \mathscr{S})$ be a continuous linear projection.

(a) Let $X_{a} ; \alpha \in \Lambda$ be components of $X$. Since $X$ is locally connecterl, $X_{a}$ are closed-open in $X$ and hence $R\left(X_{a}\right)$ are closed-open in $R(X)$ and $R(X)$ $=\bigcup_{1} R\left(X_{\alpha}\right)$. Thus wo have

$$
H^{0}(X, \mathscr{P})=\prod_{\alpha} H^{0}\left(X_{a}, \mathscr{P}\right) \quad \text { and } \quad O_{\xi}^{\infty}(R(X))=\prod_{\alpha} O_{\xi}^{\infty}\left(R\left(X_{u}\right)\right) .
$$

For each $a$ let $i_{\alpha}$ denoto the canonical embedding of $V_{\xi}^{\infty}\left(K\left(X_{u}\right)\right)$ into $\prod_{\beta} O_{\xi}^{\infty}\left(R\left(X_{\beta}\right)\right)$. Then the formula

$$
P_{a} \sigma=\left(P i_{\alpha} \sigma\right) \mid X_{a} \quad \text { for } \quad \sigma \in O_{\xi}^{\infty}\left(R\left(X_{\alpha}\right)\right)
$$

defines a continuous linear projection of $O_{\xi}^{\infty}\left(R\left(X_{\alpha}\right)\right)$ onto $H^{0}\left(X_{u}, \mathscr{S}^{p}\right)$.

(b) By (a) we can assume $X$ is connected. Hence by the local irroducibility of $X$ it is easy to see that $H^{0}(X, \mathscr{S})$ has a continuous norm $\varrho$. Consider the map $\pi_{0} P: C_{\xi}^{\infty}(R(X)) \rightarrow H^{0}(X, \mathscr{S}) / \varrho$, where $\pi_{0}: H^{0}(X, \mathscr{S})$ $\rightarrow H^{0}(X, \mathscr{S}) / \varrho$ is the canonical map. Since $O_{\xi}^{\infty}(R(X))=\lim \left\{O_{\xi}^{\infty}(G) ; G\right.$ is open in $R(X)\}$, it follows that there exists a relatively compact opon sot $G$ in $R(X)$ and a linear map $Q: \operatorname{Im} R(X, G) \rightarrow H^{0}(X, \mathscr{S}) / \varrho$ such that

$$
\pi_{0} P=Q R(X, G),
$$

where $R(X, G): C_{\xi}^{\infty}(R(X)) \rightarrow C_{\xi}^{\infty}(G)$ is the restriction map. Lot $\tilde{G}$ bo a rolatively compact neighbourhood of $\bar{G}$ in $R(X)$ and let $\varphi \in 0^{\infty}(R(X)), \varphi \mid G=1$, $\operatorname{supp} \varphi \subset \tilde{G}$. Define a continuous linear map $\hat{\varphi}: C_{\xi}^{\infty}(\tilde{G}) \rightarrow C_{\xi}^{\infty}(R(X))$ by multiplication by $\varphi$. Note that

$$
R(X, G) \hat{\varphi}=R(\tilde{G}, G) .
$$

Let $\sigma \in H^{0}(X, \mathscr{S})$. Then by (2.1) and (2.2) we have

$$
\begin{aligned}
\pi_{Q} P \hat{\varphi} R(X, \tilde{G}) \sigma & =Q R(X, G) \hat{\varphi} R(X, \tilde{G}) \sigma=Q R(\tilde{G}, G) R(X, \tilde{G}) \sigma \\
& =Q R(X, G) \sigma=\pi_{Q} P \sigma=\pi_{Q} \sigma .
\end{aligned}
$$

Since $\pi_{0}$ is injective, wo havo

$$
P \hat{\varphi} R(X, G) \sigma=\sigma \quad \text { for } \quad \sigma \in H^{0}(X, \mathscr{P}) \text {. }
$$

Let $W$ be a relatively compact neighbourhood of $\overline{\tilde{G}}$ in $R(X)$ and lot $\varrho w$ be a continuous norm on $H^{0}(X, \mathscr{P})$ defined by $\bar{W}$. Then the canonical map $q: H^{0}(X, \mathscr{S}) / \varrho_{\bar{W}} \rightarrow C_{\xi}^{\infty}(\tilde{G})$ is continuous. By (2.3) we have

$$
P \hat{\varphi} q \pi_{Q \bar{W}}=\mathrm{id} .
$$

Ironce the identity map of $H^{0}(X, \mathscr{S})$ is factorized through a Banach space. Thus by the nucloarity of $H^{\prime}(X, \mathscr{S})$ wo infer that $\operatorname{dim} H^{0}(X, \mathscr{S})<\infty$.

THEOntam 2.3. Let $X$ be a Stein space and let $\xi$ be a holomorphic Banach bundle over $X$. Then $\mathcal{O}_{\xi}(X)$ is complemented in $C_{\xi}^{\infty}(R(X))$ if and only if $X$ is discrete.

Proof. Isot $P: O_{\xi}^{\infty}(R(X)) \rightarrow O_{\xi}(X)$ bo a continuous linear projection and lot $W$ be an irreducible branch of $X$. It suffices to show that $W=\left\{z_{W}\right\}$ since $W$ is irreducible, $\mathcal{O}_{\xi}(W)$ has a continuous norm. Applying the proof of Lomma 2.2 to tho map $R P: O_{\xi}^{\infty}(R(X)) \rightarrow \mathcal{O}_{\xi}(X)$, wo get a relatively compact opon sot $Q$ in $R(X)$ and a continuours linear map $Q: O_{\xi}^{\infty}(G) \rightarrow \mathcal{O}_{\xi}(W)$ such that

$$
Q R\left(X^{*}, G^{*}\right)=\mathcal{R} \sigma \quad \text { for all } \sigma \in \mathcal{O}_{\xi}(X),
$$

where $R: \mathcal{O}_{\xi}(X) \rightarrow \mathcal{O}_{\xi}(W)$ is the restriction map. Note that $R$ is surjective ([13], Theorem 3.9). Lot $\tilde{G}$ be a relatively compact neighbourhood of $\bar{G}$ in $R(X)$ and lot $\left.q: \widehat{\mathcal{O}_{\xi}} \widehat{(X)}\right) / \varrho_{\tilde{G}} \rightarrow C_{\xi}^{\infty}(G)$ be the restriction map. Then

$$
Q q \pi_{0} \tilde{\tilde{G}}=R \sigma \quad \text { for } \quad \sigma \in \mathcal{O}_{\xi}(X) .
$$

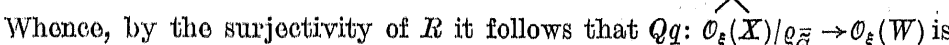
surjective. Tence by the open mapping theorom it follows that $W=\left\{z_{W}\right\}$ if $\xi$ is finito-dimensional.

Assume now that $\xi$ is infinite-dimensional. By ([13], Theorem 3.13) there exists a $\sigma \in \mathcal{O}_{\xi}(X)$ such that $\sigma(z) \neq 0$ for every $z \in X$. Considering a 1-dimonsional subbundle $\eta$ of $\xi$ generated by $\sigma(X)$. By ([13], Theorem 3.11) there exists a projection $\pi$ of $\xi$ onto $\eta$. It is easy to see that the formula

$$
(\tilde{P} \sigma) z=\pi(P \sigma(z)) \quad \text { for } \quad \sigma \in O_{\eta}^{\infty}(R(X))
$$

defines a continuous linear projection of $\sigma_{\eta}^{\infty}(R(X))$ onto $\mathcal{O}_{\eta}(X)$. This implies that $X$ is discrete.

Tho theorem is proved.

Romark 2.4. Whon $X$ is an open Stoin sot in $C^{n}$ and $\xi$ is trivial, Theorom 2.3 has beon proved by Poly [10].

8 3. The splitness of Dolbeant complexes of holomorphic Banach bundles. Lut $X$ bo a paracompact complex manifold and let $\xi$ bo a holomorphic Banach bundle over. $X$. For each $q \geqslant 0$ by $\Omega_{\xi}^{q}$ we denote the sheaf of. germs of $0^{\infty}$-forms of bidegree $(0, q)$ on $X$ with values in $\xi$. Wo write $\Omega^{q}=\Omega_{\theta}^{q}$ whore $C$ is a trivial bundle over $X$ with fibre $C$. By the Dolbeaut lemma and by the nuclearity of spaces $\Omega^{\alpha}(U)$, where $U$ are open sets in $X$, it follows that the sequence

$$
0 \rightarrow \mathcal{O}_{\xi} \rightarrow \Omega_{\xi}^{0} \stackrel{\tilde{o}_{\xi}^{0}}{\rightarrow} \Omega_{\xi}^{1} \stackrel{\tilde{o}_{\xi}^{1}}{\longrightarrow} \ldots
$$


is exact. The complex of global sections of (3.1)

$$
D(\xi): 0 \rightarrow \mathcal{O}_{\xi}(X) \rightarrow \Omega_{\xi}^{0}(X) \stackrel{\hat{\bar{\theta}}_{\xi}^{0}}{\longrightarrow} \Omega_{\xi}^{1}(X) \stackrel{\hat{\partial}_{\xi}^{1}}{\longrightarrow} \cdots
$$

is called the Dolbeaut complex of $\xi$ on $X$.

We say that the complex $D(\xi)$ splits at $p$ if there exists a continuous linear map $\gamma_{p}: \operatorname{Im} \hat{\bar{\partial}}_{\xi}^{p} \rightarrow \Omega_{\xi}^{p}(X)$ such that $\hat{\ddot{\partial}} p \gamma_{p}=\mathrm{iil}$.

We prove the following

Theorear 3.1. Let $\xi$ be a holomorphio Banach bundle over a complen manifold $X$ which is an increasing union of open Stein sets. Then $I)(\xi)$ splits at $p$ if and only if $p>0$.

The proof of Theorem 3.1. is based on the following

LeMrMa 3.2 (Corollary 5.1 [7]). Let

$$
0 \rightarrow\left\{G_{n}, \beta_{n}^{m}\right\} \stackrel{\left\{f_{n}\right\}}{\longrightarrow}\left\{F_{n}, \omega_{n}^{m}\right\} \stackrel{\left\{o_{n}\right\}}{\rightarrow}\left\{E_{n}, \alpha_{n}^{m}\right\} \rightarrow 0
$$

be a complex of projective systems of Fréchet spaoes and let $\operatorname{Kor} f_{n}=0, \operatorname{Im} f_{n}$ $=\operatorname{Ker} g_{n}, \operatorname{Im} g_{n}=E_{n}$ and $\overline{\operatorname{Im} \omega_{n+1}^{n}}=F_{n}$ for $n \geqslant 1$. Then the following conditions are equivalent:

(i) $\lim g_{n}$ is surjective.

(ii) For each $n_{0}$ there exists an $n\left(n_{0}\right) \geqslant n_{0}$ such that

$$
\operatorname{Im} \beta_{n}^{n_{0}} \text { is dense in } \operatorname{Im} \beta_{n\left(n_{0}\right)}^{n_{0}} \text { for } \quad n \geqslant n\left(n_{0}\right) .
$$

(iii) For each $n_{0}$ there exists an $n\left(n_{0}\right) \geqslant n_{0}$ such that

(3.4) The canonical map $\beta_{n_{0}}: \lim \left\{G_{n}, \beta_{n}^{m}\right\} \rightarrow G_{n_{0}}$ has a dense image in $\operatorname{Im} \beta_{n}^{n_{0}}$ for every $n \geqslant n\left(n_{0}\right)$.

We need the following.

Let $L$ be a quasi-complete locally convex space. Consider the sequence

$$
0 \rightarrow \mathcal{O}_{\xi} \mathscr{E} L \rightarrow \Omega_{\xi}^{0} \mathscr{E} L \rightarrow \Omega_{\xi}^{1} \mathscr{E} L \rightarrow \ldots,
$$

which is obtained by $\in$-tonsoring the sequence (3.1) with $L$. By the association of $\in$-product and by ([6], Lemma 1.7) the sequence (3.5) is exact. Since the sheaf $\Omega_{\xi}^{q} \mathscr{E} L$ is fine for every $q \geqslant 0$, we get

$$
H^{\alpha}\left(X, \mathcal{O}_{\xi} \in L\right)=\operatorname{Ker} \hat{\vec{\partial}}_{\xi}^{q} \& I / \operatorname{Tm}\left(\hat{\partial}_{\xi}^{q-1} \in \mathrm{i}(1)\right.
$$

for every $q \geqslant 1$.

LemMa 3.3. Let $X$ and $\xi$ be as in Theorem 3.1 and let $I$ be a quasicomplete locally convex space. Then

$$
H^{q}\left(X, \mathcal{O}_{\xi} \in L\right)=0 \text { for every } q \geqslant 2 .
$$

Proof. Let $\left\{X_{n}\right\}$ be an increasing exhaustion sequence of $X$ consisting of open Stein sets in $X$. We write $X=\bigcup_{n=1}^{\infty} W_{n}$, where $W_{n}$ is a relatively compact open sot in $X_{n}$ such that

$$
W_{n} \subset \hat{W}_{n}=\mathcal{O}\left(X_{n}\right)-\operatorname{hull}\left(W_{n}\right) \quad \text { for every } n \geqslant 1 .
$$

Sinco overy holomorphic Banach bundle over a Stein space is complemented in some tirivial. Banach bundle and since

$$
H^{a}\left(\hat{W}_{n}, \mathcal{O} \in L\right)=0 \text { for overy } q \geqslant 2 \text { and for every } n \geqslant 1
$$

([2], Theorem .13) similarly to [6] we get xelation (3.7).

Proof of Theorom 3.1. Wo can assume that $X$ is connected.

(i) Let $p>0$. Applying Lomma 3.3 and the relation (3.6) to $L$ $=\left[\operatorname{Im} \hat{\partial_{\xi}^{p}}\right]_{0}^{\prime}=\left[\operatorname{Ker} \hat{\partial_{\xi}^{p+1}}\right]_{c}^{\prime}$, we havo

$$
\operatorname{Im}\left(\hat{\partial} \hat{\partial}_{\xi}^{p} \in \mathrm{id}\right)=\operatorname{Im} \hat{\hat{\partial}} \hat{\xi}_{\xi}^{p} \in L .
$$

Since $\operatorname{Im} \hat{\hat{\partial}}_{\xi}^{\prime \prime}$ is Fréchet, it follows that $\left[\left(\operatorname{Im} \hat{\bar{\partial}}_{\xi}^{p}\right)_{c}^{\prime}\right]_{c}^{\prime}=\operatorname{Im} \hat{\hat{\partial}_{\xi}^{p}}$ and hence the indentity map id: $\operatorname{Im} \hat{\partial}_{\xi}^{p} \rightarrow \operatorname{Im} \hat{\vec{\partial}}_{\xi}^{p}$ belongs to $\operatorname{Im} \hat{\partial}_{\xi}^{p} \in L$. Hence by (3.8) we infor that $D(\xi)$ splits at $p$.

(ii) Assumo now that $D(\xi)$ splits at 0 . Then $\mathcal{O}_{\xi}(X)$ is complemented in $\Omega_{\xi}^{0}(X)$ and $\operatorname{Im} \hat{\hat{\partial}_{\xi}^{0}}$ is closed. Note that $\hat{\hat{\partial}_{\xi}^{0}}=\lim \hat{\hat{\partial}_{\xi}^{0}}$, where $\xi_{n}=\xi \mid X_{n}$. Since the map $\hat{\partial}_{\xi_{n}}^{0}: \Omega_{\xi}^{0}\left(X_{n}\right) \rightarrow \operatorname{Ker} \hat{\vec{\partial}}_{\xi_{n}}^{1}$ is surjective, we infer that $\operatorname{Im} \hat{\bar{\partial}}_{\xi}^{0}$ is dense in $\operatorname{limKer} \hat{\partial}_{\xi_{n}}^{1}$. Fence the map $\lim \hat{\bar{\partial}}_{\xi_{n}}^{0}: \lim \Omega_{\xi}^{0}\left(X_{n}\right) \rightarrow \lim \operatorname{Ker} \hat{\bar{\partial}}_{\xi_{n}}^{1}$ is surjective. Thus by Iomma 3.2 it follows that the restriction maps $R_{\alpha}$ : $\mathscr{O}_{\xi}(X) \rightarrow \mathcal{O}_{\xi}\left(X_{q}\right)$ and $R_{q}^{p}: \mathscr{O}_{\xi}\left(X_{q}\right) \rightarrow \mathcal{O}_{\xi}\left(X_{p}\right)$ satisfy condition (3.4). Let $P: \Omega_{\xi}^{0}(X)$ $\rightarrow \mathcal{O}_{\xi}(X)$ be a continuous linear projection. Since $\mathcal{O}_{\xi}(X)$ has a continuous norm, as in the proof of Lemma 2.2 the projection $P$ is written in the form

$$
P=Q R\left(X, X_{n}\right) \text { for some } n,
$$

whore $Q: \Omega_{n}^{0}\left(X_{n}\right) \rightarrow \mathcal{O}_{\xi}(X)$ is a continuous linear map. Let $q \geqslant n$ be such that

$$
\overline{\operatorname{Im} K_{\alpha}^{n}}=\overline{\operatorname{Im} R_{n}}
$$

Put

$$
\tilde{X} \cup\left\{W ; W \text { is a component of } \boldsymbol{X}_{a}, W \cap X_{n} \neq \varnothing\right\} \text {. }
$$

Note that $\tilde{X}$ is a closed-open Stein subset of $X_{q}$ and

$$
\operatorname{Ker}\left(R_{\alpha}^{n} \mid \mathcal{O}(\tilde{X})\right)=0 .
$$


Consider the eontinuous linear map $R(X, \tilde{X}) Q R\left(\tilde{X}, X_{n}\right): \Omega_{\xi}^{0}(\tilde{X}) \rightarrow \mathcal{O}_{\xi}(\tilde{X})$. Let $\sigma \in \mathcal{O}_{\xi}(\tilde{X})$. By $(3.10)$ and since $\tilde{X}$ is closed-open in $X_{q}$, it follows that there exists a sequence $\left\{\sigma_{k}\right\} \subset \mathcal{O}_{\xi}(X)$ such that $\lim _{k} \mathbb{R}_{n} \sigma_{k}=I\left(\tilde{X}, X_{n}\right) \sigma_{\text {. }}$ Hence by (3.9) we have

$$
\begin{aligned}
\left(R(X, \tilde{X}) Q R\left(\tilde{X}, X_{n}\right) \sigma\right) \mid X_{n} & =\left(\lim _{k} R(X, \tilde{X}) Q R_{n} \sigma_{k}|| X_{n}\right. \\
& =\left(\lim _{k} R(X, \tilde{X}) P \sigma_{k l}\right) \mid X_{n} \\
& =\left(\lim _{k} R(X, \tilde{X}) \sigma_{k}\right)\left|X_{n}=\sigma\right| X_{n}
\end{aligned}
$$

Hence by (3.11) we have $R(X, \tilde{X}) Q R\left(\tilde{X}, X_{n}\right) \sigma=\sigma$ for $\sigma \in \mathcal{O}_{\xi}(\tilde{X})$. Thtus $\mathcal{O}_{\xi}(\tilde{X})$ is complemented in $\Omega_{\xi}^{\prime \prime}(\tilde{X})$. This contradics Theorem 2.3. Hence $D(\xi)$ does not split at 0 .

The theorem is proved.

Remark 3.4. When $X$ is Stein and $\xi$ is finite-dimensional, Theorem 3.1 has been established by Palamodov (Proposition 5.1 [8]).

Let $X$ be a paracompact analytic space and let $\xi$ be a holomorphic Banach bundle over $X$. The groups $H^{q}\left(X, \mathcal{O}_{\xi}\right)=\lim H^{q}\left(\mathscr{U}, \mathcal{O}_{\xi}\right)$ are ondowed with the inductive topology (where $\mathscr{U}$ is an open cover of $X$ and $H^{q}\left(\mathscr{U} ; \mathcal{O}_{\xi}\right)$ $\overline{\overline{\text { top }}} \operatorname{Ker} \delta_{\xi}^{q} / \operatorname{Im} \delta_{\xi}^{q-1}$ if $\delta_{\xi}^{q}=\delta_{\xi}^{q}(\mathscr{U}): \mathscr{C}^{q}\left(\mathscr{U}, \mathbb{O}_{\xi}\right) \rightarrow C^{q+1}\left(\mathscr{U}, \mathbb{O}_{\xi}\right)$ are the coboundary maps and the spaces $\mathscr{O}^{q}\left(\mathscr{U}, \mathcal{O}_{\xi}\right)$ are equipped with the compact-open topology. In ([11]) Silva has proved that if the space $X$ is an increasing union of open Stein sets and if $H^{1}(X, \mathcal{O})$ is Hausdorff, then $X$ is SteinThe following theorem is an extension of this result:

THEOREM 3.5. Let $X$ be an analytic space which is an increasing union of open Stein sets. Let $H^{\mathrm{I}}\left(X, \mathcal{O}_{\xi}\right)$ be Hausdorff for some non-zero holomorphio Banach bundle $\xi$ over $X$. Then $X$ is Stein.

Proof. By the result of Silva we have to show that $H^{1}(X, \mathcal{O})=0$. Lot $X$. $=\bigcup_{n=1}^{\infty} X_{n}$, where $\left\{X_{n}\right\}$ is an increasing sequence of open Stein sets. Consider the cover $\mathscr{U}=\left\{X_{n}\right\}_{n=1}^{\infty}$. Since $H^{p}\left(X_{n}, \mathcal{O}\right)=0$ for $p, n \geqslant 1$, we have

$$
H^{1}(X, \mathcal{O})=\operatorname{Ker} \delta^{1} / \operatorname{Tm} \delta^{0}
$$

and

$$
\operatorname{Ker} \delta^{1}\left(\mathscr{U}_{n}\right)=\operatorname{Im} \delta^{0}\left(\mathscr{U}_{n}\right) \text { for } n \geqslant 1
$$

where $\mathscr{U}_{n}=\left\{X_{1}, \ldots, X_{n}\right\}$. Note that $\delta^{0}(\mathscr{U})=\lim \delta^{0}\left(\mathscr{U}_{n}\right)$. Thus by $(3.1,1)$ and (3.12) and by Lemma 3.2 it suffices to chock that the restriction maps $R_{q}^{p}: \mathcal{O}\left(X_{q}\right) \rightarrow \mathcal{O}\left(X_{p}\right)$ satisfy condition (3.3).

Since $H^{1}\left(X, O_{\xi}\right)$ is Hausdorff and since $\mathscr{U}$ is a Leray cover for $\mathcal{O}_{\xi}$, it follows that the canonical map $H^{1}\left(\mathscr{U}, \mathcal{O}_{\xi}\right) \rightarrow H^{1}\left(X, \mathcal{O}_{\xi}\right)$ is bijective. Honce $H^{1}\left(\mathscr{U}, \mathcal{O}_{\xi}\right)$ is Hausdorff. Thus $\operatorname{Im} \delta_{\xi}^{0}(\mathscr{U})$ is closed. Combining this with the surjectivitiy of the 1naps $\delta_{\xi_{n}}^{0}: \theta^{0}\left(\mathscr{U}_{n}, \mathscr{O}_{\xi}\right) \rightarrow \operatorname{Ker} \delta_{\xi_{n}}^{1}$, where $\xi_{n}=\xi \mid X_{n}$, we infer thit the map lini $\delta_{\xi_{n}}^{0}: \lim 0^{0}\left(\mathscr{U}_{n}, \mathcal{O}_{\xi}\right) \rightarrow \lim K e r \delta_{\xi_{n}}^{1}$ is surjective: Hence the restriction mans $\mathcal{K}_{\xi q}^{p}: \mathcal{O}_{\xi}^{\leftarrow}\left(X_{q}\right) \rightarrow \mathcal{O}_{\xi}\left(X_{p}\right)$ satify condition (3.3).

(a) Wo first assume that $\xi$ is infinite-dimensional. Given $n_{0}$. Take $q\left(n_{v_{0}}\right) \times n_{0}$ such that (3.3) holds for $\beta_{q}^{p}=R_{\xi q}^{p}$. Let $q \geqslant q\left(n_{0}\right)$. By [13] we find wu $\sigma \in \mathcal{O}_{\xi}\left(X_{q}\right)$ such that $\sigma(z) \neq 0$ for $z \in X_{q}$. Let $\eta$ be a subbundle of $\xi \mid X_{q}$ spranned by $\sigma\left(X_{q}\right)$ and lot $\theta: C \rightarrow \eta$ be a canonical isomorphism.

$$
O(\approx, \lambda)=\lambda \sigma(z) \text { for } \quad(z, \lambda) \in X_{\alpha} \times C .
$$

By (3.3) and since $\eta$ is complemented in $\left.\xi\right|_{X_{q}}$ it follows that for each $a \in$ $\mathcal{O}\left(X_{\eta\left(n_{1}\right)}\right)$ thesese (sxistis as sequence $\left\{\sigma_{n}\right\} \subset \mathcal{O}_{\eta}\left(X_{q}^{a}\right)$ such that $\sigma_{n}\left|X_{n_{0}} \rightarrow \theta \alpha\right| X_{n_{0}}$. Hencen ${ }^{-1} \sigma_{n}\left|X_{n_{0}} \rightarrow \alpha\right| X_{n_{0}}$ and thus the case where $\xi$ is infinite-dimensional is peroverl.

(1) Assume now that $\xi$ is finite-dimensional. Consider the infinitedimensional holomorphie Banach bundle $\xi \mathscr{E} B$, where $B$ is some infinitedimensional Banach space. Note that

$$
\operatorname{Kor}\left(\delta_{\xi}^{1} \mathscr{E} \mathrm{id}: O^{0}\left(\mathscr{U}, \mathcal{O}_{\xi \mathscr{G} B}\right) \rightarrow O^{1}\left(\mathscr{U}, \mathcal{O}_{\tilde{\xi} B}\right)\right)=\operatorname{Ker} \delta_{\xi}^{1} \mathscr{E} B .
$$

Hence by the nuclearity of the space $\mathscr{O}^{0}\left(\mathscr{U}, \mathcal{O}_{\xi}\right)$ it follows that $H^{1}\left(\mathscr{U}, \mathcal{O}_{\xi \delta B}\right)$ $=0$. combining this with (a), wo infer that $X$ is Stein.

The theorem is proved.

Remark 3.6. Fornuess has constructed a complex manifold which is in increstsing union of open stein sets such that $\operatorname{dim} O(F)=1$ ([3]). From Theorems 3.1 and 3.5 wo obtain an extra property of $F$.

Proposrtron 3.7. Let $\xi$ be a non-zero holomorphic Banach bundle over $F$. Ther,

(i) $D(\xi)$ splits only at $p>0$.

(ii) $H^{1}\left(X, \mathcal{O}_{\xi}\right)$ is uncountable-dimensional and is not Hausdorff.

Proof. (i.) follows from Theorem 3.1. Theorem 3.6 implies that $H^{1}\left(X, \mathcal{O}_{\xi}\right)$ is not ICausdorff. The non-countability follows from (Theorem $1.4,[12])$.

Remark 3.8. The necessity of the condition of Theorem 3.1 follows also from Theorems 2.3 and 3.5 .

\section{References}

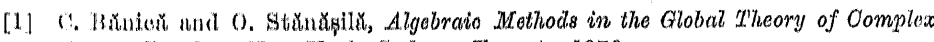
spuese, Xemilon, Now York, Sydney, Toronto 1970.

[2] 1. Bungurti, Holomorphio funotions with values in locally convex spaces and applisations to integral formulas, Trans. Amer. Math. Soc. 1.11 (1964), 317-343.

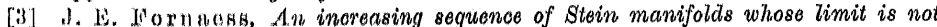
s'lein, Math. Ann, 223, 275-277.

[4] I. Gunning and II. Rossi, Analytio Ifunotions of Several Oomplex Variables, Prentico-Fall, Lnglowood Cliffs, N.J. 1965. 
[5] R. Narasimhan, Introduction to the Theory of Analytic Spaces, Tecturo Notcs in Math. 25, Springer-Verlag, Berlin-Heidelberg-New York 1966.

[6] Nguyen $\operatorname{van} \mathrm{Khue}$, On the cohomology groups of $\mathscr{S} \in L$, Studia Math. 72 (1982), 183-197.

[7] P. V. Palamodor, Homological methods in theory of locally convex spaces, Uspokhi Mat. Nauk 1 (1971), 3 64.

[8] - On Stein manifolds the Dolbeaut complexes split at positive dimensions, Mat. Sb. 88 (1972), 287-315.

[9] A. Pietsch, Nuclear Locally Convex Spaces, Akademio Vorlag, Berlin 1972.

[10] J. B. Poly, Sur opérateurs differentiels et les morphismes directes, C. R. Acad. Sci. Paris 270 (10) (1970), 647-649.

[11] A. Silv a, Rungescher satz and a condition for Steiness for the limit of an increasing sequence of Stein spaces, Ann. Inst. Fourior 28 (1978), 187-200.

[12] Y. T. Siu, Non-countable dimension of cohomology groups of analylio sheaves and domains of holomorphy, Math. Z. 102 (1967), 17-29.

[13] M. G. Zajdenberg, S. G. Krejn, P. A. Kusment, A. A. Pankov, Banteh bundles and linear operators, Uspekhi Mat. Nauk 5 (1975), 1.01-1.157.

INSTITUTE OF M.ATHEMATTOS

POLISH ACADTMYY OF SCIENCES

al. Sniadeckich 8, 00-950 Wargzawa

\section{On functions with bounded mixed variance}

by

UMB R RTO NERI (College Park, Md.)

Abstract. The eoncept of bounded mixed variance (BMV), which extends the notion of bounded mean oscillation (BMO), is discussed. The main result is that spaces of functions with bounded mixed variance are duals of certain "atomic $H^{1}$ spaces".

Introduction. In this note, we discuss a measure-theoretic concept which extends the notion of bounded mean oscillation described by John and Nirenberg in [11]. This concept, which we call bounded mixed varianoe generalizes the $\mathscr{L}^{p, \lambda}$ spaces of G. Stampacchia (see [1] and [13]), the BMO $(\varrho)$ space of [10], and the duals of weighted Hardy spaces $H^{1}$ in [14], [15], [9], [12] and [8]. To emphasize the wide applicability of our remarks, we shall place them in the setting of a space $X$ of homogeneous type, [3] and [4]. Howover, our main motivation and emphasis comes from the case $X=O D$, where $D$ is a simply-connected bounded domain in $\boldsymbol{R}^{n}$ which is a Lipschitz or $O^{1}$ domain (see Remark 1.7 below).

I thank Professors E. Fabes and $\mathrm{C}$. Kenig for their encouragement and interest in this subject.

\$ 1. Atomic spaces and preliminaries. Let $X=(X, d)$ be a space of homogeneous type (cf. [4], §2) equipped with a pair of regular Borel measures $\mu$ and $\nu$, mutually absolutely continuous and satisfying the doubling condition. That is, if $B=B_{r}(x)=\{y \in X: d(x, y)<r\}$ and $B^{*}$ $=B_{2 r}(\infty)$, then $\mu\left(B^{*}\right) \leqslant A \mu(B)$ and $\nu\left(B^{*}\right) \leqslant A \nu(B)$ for some constant $A$ independeut of the ball $B$.

Dempinixton 1.0. Let $1<q \leqslant \infty$. A function $a(x)$ is a $(1, q)$ atom of type $(\mu, \nu)$ if its support is contained in some ball $\mathbf{B}$ and

$$
\begin{gathered}
\left\{\mu(B)^{-1} \int_{B}|a|^{\alpha} d \mu\right\}^{1 / \alpha} \leqslant \mu(B)^{-1}, \\
\int a d \nu=0 .
\end{gathered}
$$

Naturally, (i) is intended for the smallest ball $B$ containing the support of $a(x)$ and the left-side of (i) equals the norm of $a$ in $L^{\infty}(X, d \mu)$ if $q=\infty$. When $\mu(X)<\infty$, the constant $\nu(X)^{-1}$ is also considered to be an atom. 